\title{
Variation in Habitat Choice and Delayed Reproduction: Adaptive Queuing Strategies or Individual Quality Differences?
}

Martijn van de Pol, ${ }^{1,2,3}{ }^{\star}$ Ido Pen, ${ }^{1}$ Dik Heg, ${ }^{2,4}$ and Franz J. Weissing ${ }^{1}$

1. Theoretical Biology Group, University of Groningen, Groningen, The Netherlands;

2. Animal Ecology Group, University of Groningen, Groningen, The Netherlands;

3. Centre for Conservation Biology, Norwegian University of Science and Technology, Trondheim, Norway; and Dutch Centre for Avian Migration and Demography, Heteren, The Netherlands; 4. Department of Behavioural Ecology, University of Bern, Bern, Switzerland

Submitted December 1, 2006; Accepted April 12, 2007;

Electronically published August 9, 2007

Online enhancements: appendixes.

ABSTRACT: In most species, some individuals delay reproduction or occupy inferior breeding positions. The queue hypothesis tries to explain both patterns by proposing that individuals strategically delay breeding (queue) to acquire better breeding or social positions. In 1995, Ens, Weissing, and Drent addressed evolutionarily stable queuing strategies in situations with habitat heterogeneity. However, their model did not consider the non-mutually exclusive individual quality hypothesis, which suggests that some individuals delay breeding or occupy inferior breeding positions because they are poor competitors. Here we extend their model with individual differences in competitive abilities, which are probably plentiful in nature. We show that including even the smallest competitive asymmetries will result in individuals using queuing strategies completely different from those in models that assume equal competitors. Subsequently, we investigate how well our models can explain settlement patterns in the wild, using a long-term study on oystercatchers. This long-lived shorebird exhibits strong variation in age of first reproduction and territory quality. We show that only models that include competitive asymmetries can explain why oystercatchers' settlement patterns depend on natal origin. We conclude that predictions from queuing models are very sensitive to assumptions about competitive asym-

* Corresponding author; e-mail: m.van.de.pol@bio.ntnu.no.

Am. Nat. 2007. Vol. 170, pp. 530-541. (c) 2007 by The University of Chicago. 0003-0147/2007/17004-42250\$15.00. All rights reserved.

DOI: $10.1086 / 521237$ metries, while detecting such differences in the wild is often problematic.

Keywords: age of first reproduction, conditional strategies, evolutionarily stable strategy, habitat selection, Haematopus ostralegus, natal habitat preference.

In many species, individuals delay reproduction beyond the age of sexual maturity (e.g., Charnov 1991; Newton 1998). Life-history theory tries to explain delayed reproduction, with explanations falling into two major classes or a combination thereof (e.g., Stearns 1992). First, young adults may delay reproduction until later stages in life to maximize lifetime reproductive output, for example, because reproducing early in life may be at the cost of reduced life span, future reproduction, or somatic growth (e.g., Oli et al. 2002; Krüger 2005). Second, delayed reproduction may be phenotype dependent, for example, because low-quality individuals may need to make the best of a bad job and are forced to delay their onset of reproduction.

Another ubiquitous pattern in nature is that once individuals reproduce, some individuals are apparently willing to do so in inferior breeding positions, such as lowquality territories, inferior positions in a colony or lek, or subdominant positions in a group. Again, two major explanations, or a combination of both, have been put forward to explain these facts (e.g., Fretwell 1972). First, occupying an inferior breeding position may maximize lifetime reproductive success when it is counterbalanced by a longer life span. Second, certain phenotypes may be forced to occupy low-quality breeding opportunities; that is, they are despotically excluded by others from the best breeding spots.

Recently, an alternative hypothesis has been put forward that tries to simultaneously explain both variation in age of first reproduction and variation in settlement decisions. The queue hypothesis suggests that individuals maximize lifetime fitness by strategically waiting (queuing) for high- 
quality breeding opportunities to become available, instead of immediately accepting a low-quality breeding position (Zack and Stutchbury 1992; Ens et al. 1995). Moreover, the queue hypothesis does not necessarily invoke the existence of individual quality differences (i.e., some individuals are competitively inferior and therefore have to make the best of a bad job), although, of course, the queue hypothesis and the individual quality hypothesis do not necessarily exclude each other.

Originally, queuing was a shorthand term to describe how individuals delay reproduction and stay within cooperatively breeding groups to inherit the dominant breeding position (Wiley and Rabenold 1984). Nowadays, it is realized that queuelike systems range from queues for mating opportunities (Schwagmeyer and Parker 1987), social and breeding position in group-living and cooperativebreeding species (Wiley and Rabenold 1984; East and Hofer 2000; Heg et al. 2005; Mitchell 2005), positions on the lek (Kokko et al. 1998), or access to harems or colonies (Poston 1997; Voigt and Streich 2003) to queues for highquality territories (Zack and Stutchbury 1992; Ens et al. 1995; Ekman et al. 2001). Moreover, queuing processes seem to occur in a wide variety of taxa (birds, fish, mammals, and invertebrates) and life histories.

Adaptive queuing models try to predict how much individuals should be willing to delay reproduction and which reproductive opportunity to accept (Ens et al. 1995; Kokko et al. 1998, 2001; Pen and Weissing 2000, 2001; Kokko and Ekman 2002). An important feature of queuing decisions is their frequency-dependent nature. Ens, Weissing, and Drent (Ens et al. 1995; henceforth EWD) were the first to stress - and mathematically formalize - that the success of a queuing strategy depends on how many other individuals are queuing for the same opportunity. They showed that in evolutionary equilibrium, the competition, and thereby queuing time, for high-quality breeding positions increases up to a point at which it pays to accept low-quality breeding positions at a young age. Thereby, EWD extended the ideal despotic distribution into a lifehistory framework and showed how delayed reproduction and habitat selection can be viewed as two sides of the same coin. Frequency-dependent queuing processes now have been used to explain the logic of delayed breeding (EWD), territory choice (Kokko and Sutherland 1998; Kokko et al. 2001; Pen and Weissing 2001), reproductive skew (Kokko and Johnstone 1999), and cooperative breeding (Pen and Weissing 2000; Kokko and Ekman 2002).

Although EWD developed a general queuing model, their model was also used to study territory choice and delayed reproduction in a population of free-living oystercatchers (Haematopus ostralegus), a long-lived shorebird. Oystercatchers are an interesting test case because some individuals clearly occupy better territories than oth- ers and some individuals delay breeding up to 8 years longer than others (van de Pol et al. 2006). Strikingly, in this species there seem to be no clear indications that individuals occupying high- or low-quality territories or individuals that delay or do not delay reproduction differ in their competitive abilities (EWD; also Bruinzeel et al. 2006). This puzzling observation prompted EWD to investigate whether adaptive queuing decisions could explain the large variation in territory choice and delayed reproduction in oystercatchers without invoking differences in individual quality. However, EWD could not yet provide a rigorous test of the quantitative predictions of their queue model, because it takes many years to gather sufficient field data on long-lived oystercatchers.

The aim of this article is threefold. First, we improve the original EWD model by removing an important inconsistency from the model and incorporating population limitation. These modifications result in a substantially different evolutionarily stable queuing strategy. Second, the original EWD model did not incorporate individual quality differences. However, differences in competitive abilities are probably plentiful in many species, although they sometimes may be hard to detect. To investigate the relative importance of the queue hypothesis and the individual quality hypothesis, we extend the original model to allow queuing strategies to differ between individuals and also incorporate differences in competitive abilities. Third, after 21 years of study, we are now able to perform a quantitative comparison between the predictions of these queuing models and the observed settlement behavior of oystercatchers in the field. To our knowledge, no other studies have compared predictions from adaptive queuing models with settlement patterns and variation of age of first reproduction in the wild, although such a quantitative comparison is crucial for our understanding of the importance of queuing processes in nature.

\section{The Queuing Models \\ The Rationale}

The EWD model addressed habitat choice and delayed reproduction in the simplest case of habitat heterogeneity, in which a surplus of nonbreeders $(\mathrm{N})$ compete for a limited number of high- $(\mathrm{H})$ and low-quality (L) territories. The queue hypothesis suggests that variation in habitat choice and delayed reproduction can be understood by taking the perspective of nonbreeders facing the "career decision" of when and where to settle. Because individuals cannot opt for two options with the same success (because of constraints in time and space), nonbreeders do best to choose between two strategies. In the first strategy, which we call the "QL strategy," nonbreeders queue for low- 
quality habitat and try to settle there. Individuals queuing for low-quality territories are likely to start reproducing at an early age and produce few offspring per year. In the second strategy, which we call the "QH strategy," nonbreeders wait (queue) for a high-quality territory to become available. Because competition for high-quality territories will be fierce, individuals queuing for high-quality territories $(\mathrm{QHs})$ will not reproduce before a much older age than individuals queuing for low-quality territories (QLs). Moreover, individuals queuing for high-quality territories will have a high chance of dying before ever reproducing but, if successful, will produce many offspring per year. Although the two strategies have different shortterm payoffs, the key question is whether they differ in long-term fitness. Furthermore, the success of both strategies directly depends on the frequency in the population at which nonbreeders choose to queue for either high- or low-quality territories, as this determines the strength of competition. Ens et al. (1995) argued that in evolutionary equilibrium, the proportion of nonbreeders that follows a $\mathrm{QH}$ or a QL strategy is such that the expected lifetime reproductive success is equal for both strategies. This makes intuitive sense, because if the fitness of one strategy is higher than the other, nonbreeders should switch to the strategy with higher payoff until fitness differences disappear because of intensified competition. It is important to note that the queue hypothesis takes a perspective fundamentally different from that of most other studies on delayed breeding (e.g., Oli et al. 2002; Krüger 2005 and references therein). In understanding the fitness consequences of delayed reproduction, the queue hypothesis takes into account the fate of both successful (i.e., recruited) and unsuccessful individuals (i.e., died before recruitment), while most other studies compare only the fitness of successful individuals that vary in age of first reproduction.

\section{Shortcomings of the EWD Model}

Although the general idea behind the queue hypothesis is attractive, the implementation of the model in EWD has three important shortcomings. First, EWD used a demographic model to calculate the expected lifetime reproductive success of queuers and subsequently used these results to calculate the evolutionarily stable strategy (ESS) in a separate model. However, these two models are not fully consistent. In fact, inserting the ESS into the demographic model yields a stage distribution that is inconsistent with the data and analyses on which the demographic model was based (ESS: $\mathrm{N}=60 \%, \mathrm{H}=21 \%$, $\mathrm{L}=19 \%$; demographic model: $\mathrm{N}=31 \%, \mathrm{H}=28 \%$, $\mathrm{L}=41 \%)$. Therefore, we here use an approach that integrates the population dynamics and evolutionary dy- namics of life-history strategies in one coherent model. Second, EWD did not include density regulation in their model. In fact, their ESS population increases indefinitely, which is not very realistic. Therefore, we here introduce population limitation in a natural way by setting a maximum on the number of suitable breeding territories. Third, the EWD model does not allow the investigation of the individual quality hypothesis. Ens et al. (1995) did not include individual differences in competitive abilities because there were no clear indications for such differences in oystercatchers. However, differences in competitive abilities are probably very common in many species (e.g., Stearns 1992), and it therefore seems important to incorporate them to generalize the applicability of the model. In fact, even in the population of oystercatchers described in the EWD study, we have recently shown that settlement patterns differ strongly between nonbreeders born in highand low-quality habitats (van de Pol et al. 2006). Although this is not direct evidence for the existence of differences in competitive abilities, it does suggest that queuing strategies depend on natal origin. Therefore, we extend the original model to allow for conditional queuing strategies to evolve in which individuals born in high- and lowquality territories can use different queuing strategies. In this model, we assess how competitive asymmetries (i.e., individual quality differences) between nonbreeders born in high- and low-quality territories affect adaptive queuing decisions.

\section{New Model with Unconditional Queuing Strategies}

To address the first two shortcomings, we constructed a new version of the EWD model that combines the population dynamics and life-history strategies in one coherent model and also incorporates population regulation. We refer to this model as the unconditional model (i.e., not conditional on natal origin); the role of conditional queuing strategies is investigated in the next section. Our unconditional model is based on the graphical representation in figure $1 A$. The corresponding mathematical model and ESS can be analyzed using standard methods (e.g., Taylor 1990; Caswell 2001) and are described in full technical detail in appendix A in the online edition of the American Naturalist; below, we describe the major steps and main results.

The variable $x$ represents the queuing strategy in the population; when $x=0$, all individuals queue for highquality territories; when $x=1$, all individuals queue for low-quality territories. The changes in numbers of individuals queuing for high- and low-quality territories and the numbers of high- and low-quality territory owners can be described by a system of recurrence equations based on the flows between states in figure $1 A$. For reasons of 
(A)

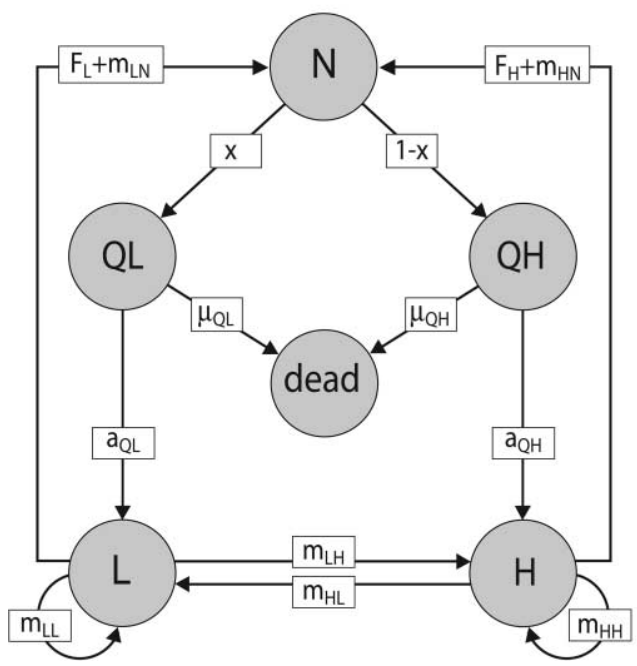

unconditional strategies

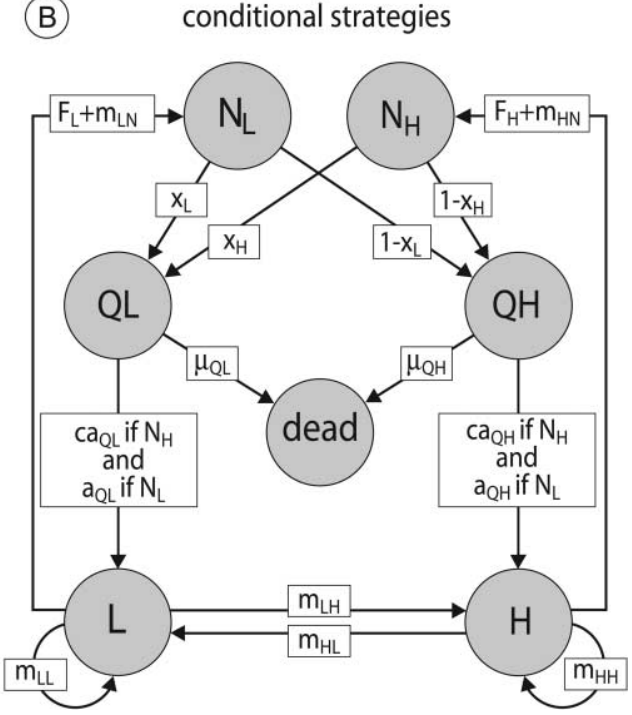

Figure 1: Schematic representation of a situation with $(A)$ unconditional queuing strategies and $(B)$ conditional queuing strategies. The parameter $x$ represents the strategic choice individual nonbreeders $(N)$ have to make to queue for either a high- or a low-quality territory $(Q H$ and $Q L$, respectively). Breeders in high- and low-quality territories ( $H$ and $L$, respectively) can produce new nonbreeders $\left(F_{\mathrm{H}}\right.$ and $\left.F_{\mathrm{L}}\right)$ as well as return to the nonbreeder state by losing their territory $\left(m_{\mathrm{HN}}\right.$ and $\left.m_{\mathrm{LN}}\right)$. In the conditional model, nonbreeders originating from high- and low-quality habitats can make a different strategic choice $\left(x_{\mathrm{H}}\right.$ and $x_{\mathrm{L}}$, respectively). Furthermore, competitive asymmetries can be included by giving nonbreeders born in high-quality territories $\left(N_{\mathrm{H}}\right)$ a $c$-times-higher annual probability of settling $\left(c a_{\mathrm{OH}}\right.$ and $\left.c a_{\mathrm{QL}}\right)$ than nonbreeders born in low-quality territories $\left(N_{\mathrm{L}}\right)$ have $\left(a_{\mathrm{QH}}\right.$ and $\left.a_{\mathrm{QL}}\right)$. Competitive asymmetries $(c)$ reflect differences in, for example, fighting capacity, are assumed to be externally given, and usually cannot be directly estimated in the field. Note that only the flows between states (arrows) that are necessary for constructing the model are presented (e.g., $L$ can die, but this is given by $\mu_{\mathrm{L}}=1-m_{\mathrm{LL}}-m_{\mathrm{LN}}-m_{\mathrm{LH}}$ ).

simplicity, several (implicit) assumptions are made in this model. First, we assume that all parameters are time, sex, and age independent. Second, we assume that the numbers of suitable high- and low-quality territories are fixed over time, thereby limiting the population (see "Discussion"). Because breeding opportunities are limited and there is a surplus of nonbreeders, the annual settlement probabilities of QHs and QLs ( $a_{\mathrm{QH}}$ and $a_{\mathrm{QL}}$, respectively) directly depend on the number of competitors, which is determined by the frequency of $x$ in the population. All other parameters are assumed to be density and frequency independent. Third, we assume that the behavior of nonbreeders with breeding experience (breeders that have lost their territory) is similar to that of nonbreeders without breeding experience (see "Discussion").

From our system of recurrence equations, we can derive the state-dependent reproductive values $v$, the relative contribution of different types of individuals to the population growth rate, which are the standard fitness measure in evolutionary cost-benefit analysis (e.g., Taylor 1990; Caswell 2001). To derive the ESS, we assessed the fitness of a mutant strategy $x$ in an established population of individuals playing strategy $x^{*}$. The ESS is obtained by finding the value of $x^{*}$ that cannot be invaded by any alternative mutant strategy, since the established population has a higher fitness than all mutants. The queuing strategy of the established population $\left(x^{*}\right)$ is implicitly contained in the annual settlement probabilities $\left(a_{\mathrm{QH}}^{*}\right.$ and $\left.a_{\mathrm{QL}}^{*}\right)$. It can be shown that coexistence of the QH and QL strategies at evolutionary equilibrium requires that their reproductive values be equal $\left(v_{\mathrm{QH}}^{*}=v_{\mathrm{QL}}^{*}\right)$, confirming the result of EWD that at the ESS, both strategies must yield equal long-term fitness payoffs. From this condition, it follows that in evolutionary equilibrium, the ratio of QHs to QLs reflects the ratio of the expected benefits of the two types of strategies (app. A):

$$
\frac{x^{*}}{1-x^{*}}=\frac{m_{\mathrm{NL}}\left(v_{\mathrm{L}}^{*}-v_{\mathrm{QL}}^{*}\right)}{m_{\mathrm{NH}}\left(v_{\mathrm{H}}^{*}-v_{\mathrm{QH}}^{*}\right)} \text {. }
$$

This result was also obtained by EWD (their eq. [15]), although they used expected future reproductive success instead of reproductive values. They subsequently calculated $x^{*}$ using realized settlement probabilities of nonbreeders $\left(m_{\mathrm{NH}}\right.$ and $\left.m_{\mathrm{NL}}\right)$. However, this approach has two important disadvantages. First, life-history parameters of nonbreeders, such as $m_{\mathrm{NH}}$ and $m_{\mathrm{NL}}$, are notoriously difficult to reliably estimate in the field because of the incomplete 
site fidelity of nonbreeders in many species. Second, the use of estimates of realized settlement probabilities from field data $\left(m_{\mathrm{NH}}\right.$ and $\left.m_{\mathrm{NL}}\right)$ to calculate the ESS is rather begging the question, as these same parameters are also used for model validation (i.e., they determine the age of first reproduction and recruitment patterns). Therefore, we took a different approach to calculating $x^{*}$ that takes full advantage of the fact that we now have incorporated population limitation in our model. Because the total number of suitable territories is assumed to be fixed, the availability of empty territories for nonbreeders (and thereby settlement probabilities) can be predicted from the behavior of the breeders (breeder mortality, territory loss, and switching between habitats). In other words, in our model, settlement probabilities are generated by the model itself, resulting in an internally consistent model, while this was not the case in the EWD model. When we define $q$ as the ratio of high- to low-quality territories and $p$ as the ratio of the total production of new nonbreeders by high-quality territory owners to that of low-quality territory owners, the ESS value for $x^{*}$ in equation (1) can be rewritten as (app. A)

$$
x^{*}=\frac{\left(1-m_{\mathrm{HH}}+m_{\mathrm{LH}} p\right)\left(1-m_{\mathrm{LL}}-m_{\mathrm{HL}} q\right)}{\left[\left(1-m_{\mathrm{HH}}\right)\left(1-m_{\mathrm{LL}}\right)-m_{\mathrm{HL}} m_{\mathrm{LH}}\right](1+p q)} \text {. }
$$

In equation (2), $x^{*}$ is expressed as a function of several life-history parameters of breeders, which can be more reliably estimated than life-history parameters of nonbreeders (as in eq. [1]).

\section{Conditional Queuing Strategies and Competitive Asymmetries}

We extended the previous model to allow nonbreeders born in both high- and low-quality habitats $\left(\mathrm{N}_{\mathrm{H}}\right.$ and $\left.\mathrm{N}_{\mathrm{L}}\right)$ to make an independent choice of which strategy to follow $\left(x_{\mathrm{H}}\right.$ and $x_{\mathrm{L}}$, respectively; figure $1 B$ ). The strategic choice that $\mathrm{N}_{\mathrm{H}}$ have to make can vary between $x_{\mathrm{H}}=0$, when all $\mathrm{N}_{\mathrm{H}}$ become QHs and we have complete natal habitat preference, and $x_{\mathrm{H}}=1$, when all $\mathrm{N}_{\mathrm{H}}$ become QLs and we have complete natal habitat avoidance. Similarly, for $\mathrm{N}_{\mathrm{L}}$, the choice varies between $x_{\mathrm{L}}=0$, when all individuals leave the habitat type they were born in, and $x_{\mathrm{L}}=1$, when all individuals return to the habitat type they were born in. In addition, we incorporated a new parameter, $c$, that reflects differences in competitive abilities between $\mathrm{N}_{\mathrm{H}}$ and $\mathrm{N}_{\mathrm{L}}$. When $c=1$, there are no competitive asymmetries; when $c>1, \mathrm{~N}_{\mathrm{H}}$ are competitively superior over $\mathrm{N}_{\mathrm{L}}$, so that they have a $c$ times higher annual probability of acquiring a territory; and the opposite holds for $c<1$.

If there are no competitive asymmetries between $\mathrm{N}_{\mathrm{H}}$ and $\mathrm{N}_{\mathrm{L}}(c=1)$, the ESS condition results in a neutral line of equilibrium conditional strategies $\left(x_{\mathrm{H}}^{*}, x_{\mathrm{L}}^{*}\right)$ given by (app. A)

$$
x_{\mathrm{L}}^{*}=\frac{\left(1-m_{\mathrm{HH}}+m_{\mathrm{LH}} p\right)\left(1-m_{\mathrm{LL}}-m_{\mathrm{HL}} q\right)}{\left(1-m_{\mathrm{HH}}\right)\left(1-m_{\mathrm{LL}}\right)-m_{\mathrm{LH}} m_{\mathrm{HL}}}-p q x_{\mathrm{H}}^{*}
$$

At the population level, the conditional strategies $x_{\mathrm{H}}^{*}$ and $x_{\mathrm{L}}^{*}$ yield an average strategy $x^{*}$ that is identical to the population strategy in the unconditional model (eq. [2]). When $c=1$, there is no selection pressure that results in one combination of $x_{\mathrm{H}}^{*}$ and $x_{\mathrm{L}}^{*}$ being favored over any other. Therefore, starting out with a population using unconditional queuing strategies $\left(x_{\mathrm{H}}^{*}=x_{\mathrm{L}}^{*}=x^{*}\right)$, it is unlikely that conditional queuing strategies evolve.

When there are competitive asymmetries between $\mathrm{N}_{\mathrm{H}}$ and $\mathrm{N}_{\mathrm{L}}(c \neq 1)$, the ESS changes substantially (see app. A for details). Asymmetric conflicts generally do not allow for a completely mixed ESS (Maynard Smith and Parker 1976). Consequently, an ESS can exist only when at least one group of individuals plays a pure strategy. There are two candidate ESSs where $\mathrm{N}_{\mathrm{H}}$ play a pure strategy, $x_{\mathrm{H}}^{*}=0$ and $x_{\mathrm{H}}^{*}=1$, and two candidate ESSs where $N_{\mathrm{L}}$ play a pure strategy, $x_{\mathrm{L}}^{*}=0$ and $x_{\mathrm{L}}^{*}=1$. Which of these four candidate equilibria is evolutionarily stable depends on the actual values of life-history parameters. Furthermore, the conditional equilibrium strategies $x_{\mathrm{H}}^{*}$ and $x_{\mathrm{L}}^{*}$ yield an average population strategy $x^{*}$ that is no longer identical to the population strategy in the unconditional model.

\section{Application of Models to Oystercatchers \\ Territory Quality and Delayed Reproduction in Oystercatchers}

From 1984 to 2004, we studied an individually marked breeding population on the Dutch Wadden Sea island of Schiermonnikoog $\left(53^{\circ} 29^{\prime} \mathrm{N}, 6^{\circ} 14^{\prime} \mathrm{W}\right)$. Oystercatchers are long-lived ( $>30$ years) and socially and genetically monogamous and exhibit high site fidelity (Heg et al. 1993, 2003). In many oystercatcher populations, there is a clear dichotomy in habitat quality caused by permanent differences in the spatial organization of territories (Ens et al. 1992; Safriel et al. 1996). Some parents_called residentshave adjacent nesting and feeding territories, which allows them to take their chicks to the food. Other parentscalled leapfrogs-have spatially separated nesting and feeding territories and are forced to bring the food to their chicks (see fig. 1 in Ens et al. 1992). Because transporting every food item to the chicks is less efficient, leapfrogs produce consistently fewer offspring per year than residents. Thus, we categorized leapfrog territories as lowquality territories and resident territories as high-quality 

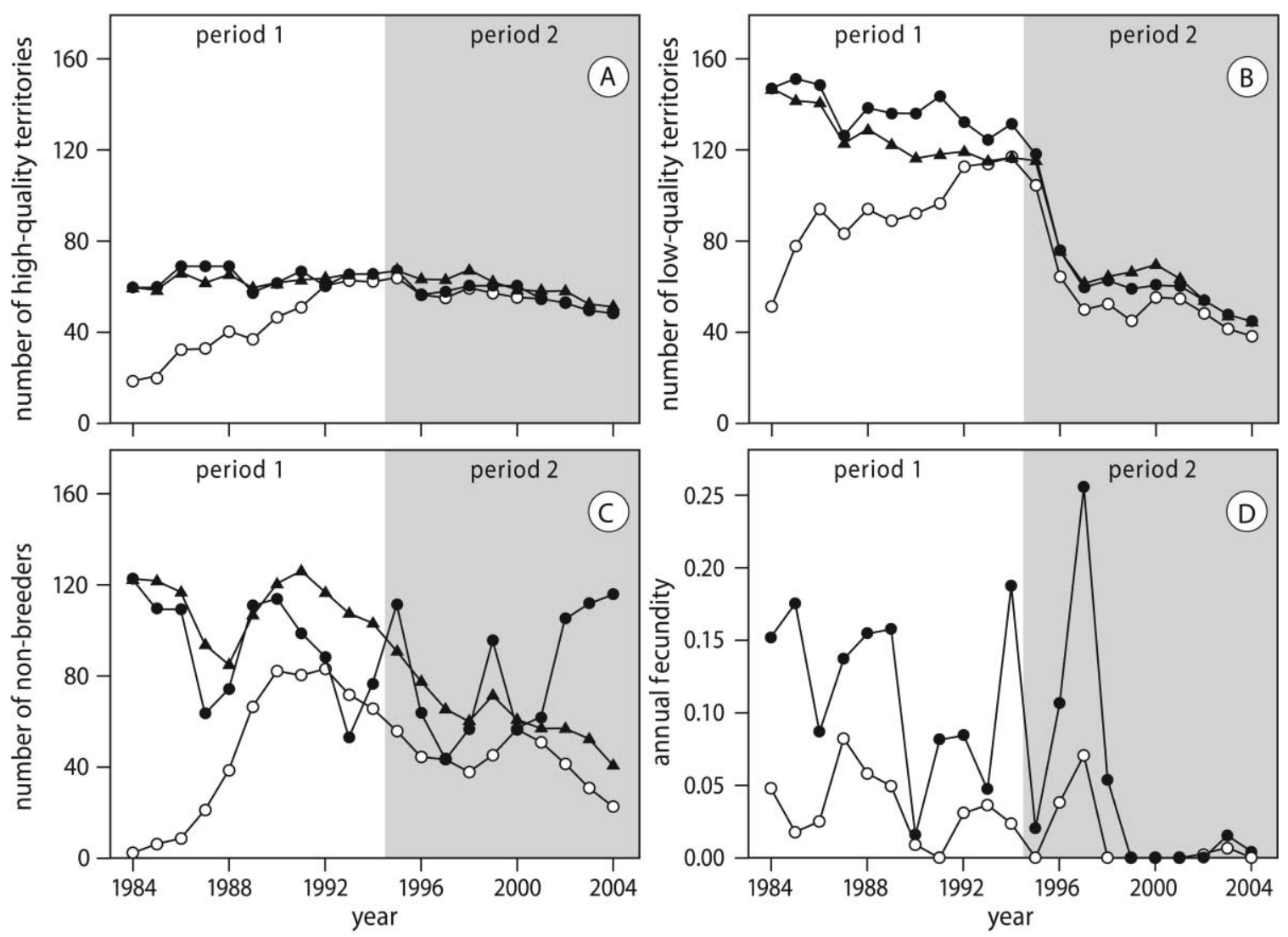

Figure 2: Changes in population numbers and fecundity from 1984 to 2004. Presented are the number of $(A)$ high-quality territories, $(B)$ lowquality territories, and $(C)$ nonbreeders. In $A-C$, filled circles refer to the counted number of individuals in each state $\left(T_{\mathrm{H}}, T_{\mathrm{L}}\right.$, and $\left.T_{\mathrm{N}}\right)$, open circles refer to the number of color-ringed individuals, and triangles refer to the number of individuals predicted by a demographic model based on estimated life-history parameters (see text). $D$, Annual fecundity in high-quality territories $\left(F_{\mathrm{H}}\right.$; filled circles) and in low-quality territories $\left(F_{\mathrm{L}}\right.$; open circles). Period 1 (1984-1994) represents a situation where the population was in equilibrium and numbers were fairly stable, while in period 2 (1995-2004), the population was declining strongly.

territories. There is a surplus of adult nonbreeders that do not defend nesting territories. Juveniles differ in plumage from adults in their first 2 years of life; therefore, we assume that oystercatchers become sexually mature and join the adult nonbreeder pool when they reach the age of 3 years. Oystercatchers show large variation in age of first reproduction (range 3-11 years). Mortality occurs mainly in winter; subsequent status changes finalize before the breeding season.

\section{Estimation of Model Parameters from Field Data}

To make quantitative model predictions, we estimated the relevant model parameters from field data. We reestimated all parameters used by EWD because we now have more years of data, data from a larger area, and better statistical estimation procedures. For technical details of estimation procedures, annual values, and a comparison with EWD estimates, see appendix B in the online edition of the American Naturalist. The number of high-quality territories $\left(T_{\mathrm{H}}\right.$; fig. $\left.2 \mathrm{~A}\right)$ was half the number of occupied lowquality territories $\left(T_{\mathrm{L}}\right.$; fig. $2 B$ ). There were substantial numbers of nonbreeders ( $T_{\mathrm{N}}$; fig. $2 C$ ), but annual counts serve only as a rough indication because sampling error was large. Annual fecundity was defined as the annual number of female offspring produced by a pair that survived until adulthood and entered the local nonbreeder pool (young are philopatric; van de Pol et al. 2006). Highquality territories consistently had three times higher annual fecundity $\left(F_{\mathrm{H}}\right)$ than low-quality territories $\left(F_{\mathrm{L}}\right)$, except in years with no young (fig. 2D). Differences in territory quality in terms of annual fecundity are thus highly predictable. Annual probabilities of status change and statedependent mortality were estimated using a multistate capture-recapture model (fig. 3). This model estimates all transition and mortality parameters simultaneously and 


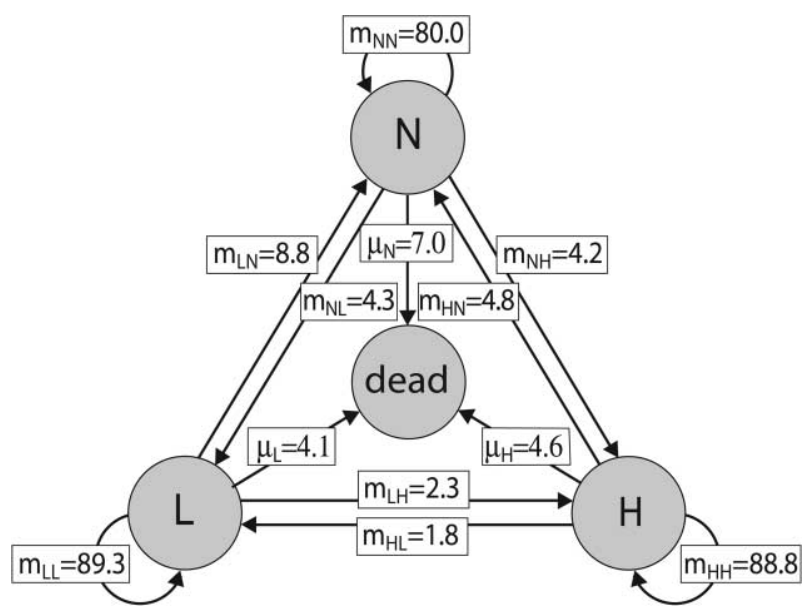

Figure 3: Annual state transition $(m)$ and mortality $(\mu)$ probabilities (\%) of owners of high- $(H)$ and low-quality territories $(L)$ and of nonbreeders $(N)$. Values were estimated using a multistate mark-recapture model and are averages for period 1 (1984-1994).

accounts for the fact that not all individuals were always resighted. Furthermore, by comparing information from local resightings with recoveries of dead individuals from a much larger area, we could adjust estimates for permanent migration.

To assess the consistency of our estimates of life-history parameters with population counts, we constructed a demographic model (see van de Pol et al. 2006). We calculated the expected change in numbers over the study period by using the numbers per state in the first year of study as initial conditions and demographic rates of each year as projection matrices. Despite the fact that we were dealing with an open population, the demographic model described the counted population numbers well, indicating that both types of model parameters are consistent with each other (fig. 2). Because population dynamics changed systematically during the 21 years of study, we distinguished two periods. In the first period (1984-1994), numbers were fairly stable, and fecundity roughly balanced mortality; during the second period (1995-2004), most low-quality territories were abandoned, and fecundity was below replacement level (fig. 2). Furthermore, life-history parameters (co-)varied between years. Therefore, besides estimating mean values over the years, we also estimated their temporal variation and covariation (table $\mathrm{B} 3$ in the online edition of the American Naturalist). Using mean values and (co)variances of all life-history and population parameters, we constructed a multivariate normal distribution of all parameters that are used in the queuing models. From this distribution, we randomly drew 1,000 sets of model input parameters, which were used to calculate confidence intervals around model predictions.

\section{Application of the Unconditional Queue Model to Oystercatchers}

We restricted the application of the queuing models to oystercatchers to period 1 (fig. 2). Using life-history parameters from period 2 in an equilibrium approach results in a situation where there are not enough new nonbreeders produced to queue for low-quality territories $\left(x^{*}=0\right)$. In addition, we assumed that there are no differential costs to the $\mathrm{QH}$ and QL strategies because prospecting behavior in oystercatchers is highly ritualized and fights are rare (Heg et al. 2000). The reason we were forced to assume that the two types of nonbreeders have similar annual mortality $\left(\mu_{\mathrm{QH}}=\mu_{\mathrm{QL}}=\mu_{\mathrm{N}}\right)$ is that in the field, it is difficult to attribute all nonbreeding oystercatchers to either the $\mathrm{QH}$ or the QL strategy.

By inserting parameter estimates averaged over period 1 in equation (2), we predict that $30 \%$ of all nonbreeders follow a QH strategy and $70 \%$ follow a QL strategy $\left(x^{*}=0.70 ; 95 \%\right.$ confidence interval: $\left.0.59-0.85\right)$. This result is exactly opposite to that of EWD, who predicted that $70 \%$ of all individuals followed a $\mathrm{QH}$ strategy $\left(x^{*}=0.30\right)$. This difference is not a consequence of differences in estimation of model parameters, because using EWD's field estimates in our model yielded $x^{*}=0.66$. Conversely, when we insert the parameters estimated in this study in the EWD model, we found $x^{*}=0.26$, confirming that differences between studies are caused by differences in modeling approach. Our results were quite robust to small deviations in mean values of model parameters (see sensitivity analysis in app. $\mathrm{C}$ in the online edition of the American Naturalist). Moreover, in contrast to the EWD model, the equilibrium stage distribution of our model fitted the proportions of nonbreeders and highand low-quality territory owners in the field well (model: $\mathrm{N}=25 \% \pm 4 \%, \quad \mathrm{H}=24 \% \pm 3 \%, \quad \mathrm{~L}=51 \% \pm 3 \%$; field: $\mathrm{N}=31 \%, \mathrm{H}=22 \%, \mathrm{~L}=47 \%$ ).

We calculated the expected life histories of individuals that queue for either a high- or a low-quality territory at evolutionary equilibrium (fig. 4). Within 3 years after sexual maturity, virtually all QLs are expected to have settled, with only a small proportion dying before ever reproducing (9\%). In contrast, most queuers for high-quality territories have to wait a long time before they can start to reproduce, and consequently QHs have a high probability of dying before ever reproducing (39\%). Although the model predicts that $70 \%$ of nonbreeders follow a QL strategy, competition is expected to be most fierce for the few high-quality territories. Individuals queuing for highquality territories not only have fewer settlement options 


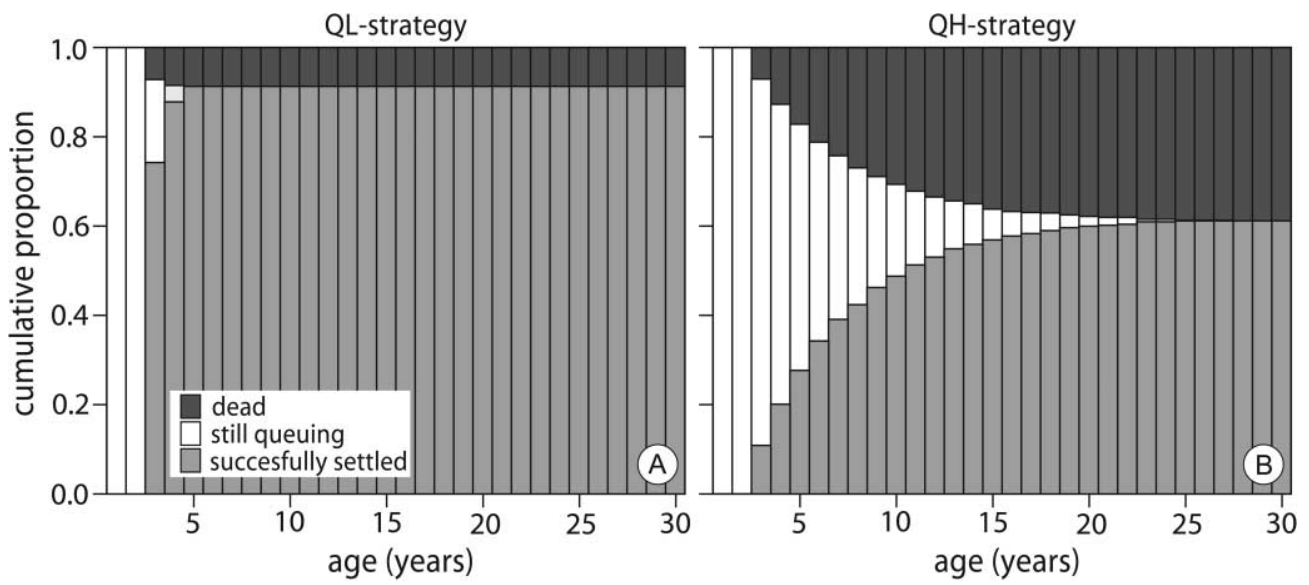

Figure 4: Predicted recruitment patterns of individuals that follow a strategy to queue for either $(A)$ low-quality territories or $(B)$ high-quality territories (unconditional model). Plotted are the changes in a cohort followed over time (i.e., age) of the proportions of (1) individuals that successfully settled, (2) individuals that are still queuing, and (3) individuals that died before ever settling. Note that we assume that oystercatchers become sexually mature when they reach the age of 3 years.

$\left(T_{\mathrm{L}} \gg T_{\mathrm{H}}\right)$ but also have to compete with the many lowquality territory owners switching to high-quality territories $\left(T_{\mathrm{L}} m_{\mathrm{LH}} \gg T_{\mathrm{H}} m_{\mathrm{HL}}\right)$. Consequently, we expect, on average, 0.56 nonbreeders actively queuing per high-quality territory, while we expect only 0.13 nonbreeders queuing per low-quality territory.

The unconditional model predicted that in equilibrium, $22 \%$ of all successful recruits first settle in a high-quality territory and $78 \%$ in a low-quality territory, which is remarkably close to observed patterns in the field $(20 \%$ and $80 \%$, respectively; table 1 , top). The unconditional model cannot explain why settlement patterns depend on natal origin in oystercatchers because it does not allow for conditional queuing strategies (see next section). Predicted age of first settlement was 4.3 years higher for QHs than for QLs (7.6 vs. 3.3 years; table 1, bottom). In the field, age of first settlement was only 1.1 year higher in high-quality territories than in low-quality territories ( 7.6 vs. 6.5 years; table 1, bottom). Although the observed difference of 1.1 years was statistically significant (ANOVA, settlement territory type: $P=.044$; sex: $P>.40$ ), it was considerably smaller than that predicted by the model. This discrepancy between model predictions and field data was due to the fact that in the field, QLs settled later than our model predicted.

\section{Application of the Conditional Queue Model to Oystercatchers}

When we assumed that there was no competitive asymmetry between nonbreeders of different natal origin $(c=1)$, the neutral line of equilibria (eq. [3]) was given by $x_{\mathrm{L}}^{*}=$
$1.38-0.98 x_{\mathrm{H}}^{*}$, which includes the solution of the unconditional model (fig. 5). When we assumed that there were asymmetries in competitive abilities $(c \neq 1)$, this line of equilibria disappeared, even for infinitesimally small asymmetries. This result shows that the assumption of no competitive asymmetries is rather specific and gives nonrobust results. For all scenarios where $\mathrm{N}_{\mathrm{H}}$ are competitively superior over $\mathrm{N}_{\mathrm{L}}(c>1)$, only one ESS exists where all $\mathrm{N}_{\mathrm{L}}$ become QLs $\left(x_{\mathrm{L}}^{*}=1\right.$; pure strategy) and $\mathrm{N}_{\mathrm{H}}$ become both $\mathrm{QHs}$ and QLs $\left(0<x_{\mathrm{H}}^{*}<1\right.$; mixed strategy). The exact value of $x_{\mathrm{H}}^{*}$ depends on how strong the competitive asymmetry is, but only weakly so (fig. 5). This weak dependence makes sense because there are so many low-quality territories that virtually all QLs can acquire a territory immediately; further increasing competitive asymmetries for a resource that is barely competed for has little additional effect. When $c<$ 1 , the situation of $x_{\mathrm{L}}^{*}=1$ is unstable, and an ESS is reached when $x_{\mathrm{H}}^{*}=1$ and $x_{\mathrm{L}}^{*}=0.40$ (for $c=0.99$; fig. 5). Therefore, inferior competitors always do best to follow a pure strategy of opting for the resource for which there is least competition. When competitive asymmetries exist $(c \neq 1)$, the average queuing strategy in the population $x^{*}$ is always higher than that in the unconditional model, but this difference is small $(<5 \%)$ for a wide range of $c$ values.

Conditional strategies, in combination with even very small differences in competitive abilities, can explain a striking feature of settlement patterns in oystercatchers. Namely, the observation that oystercatchers born in lowquality territories virtually always settled in low-quality territories (cf. pure strategy), while oystercatchers born in high-quality territories settled in both type of territories (cf. mixed strategy), is qualitatively in agreement with the 
Table 1: Comparing settlement patterns and age of first reproduction (years) of model predictions with observed values in the field

\begin{tabular}{|c|c|c|c|c|c|c|c|c|}
\hline \multirow[b]{3}{*}{ Settlement strategy } & \multirow{2}{*}{\multicolumn{2}{|c|}{$\begin{array}{c}\text { Unconditional model } \\
\text { All offspring } \\
\left(\mathrm{N}_{\mathrm{H}}+\mathrm{N}_{\mathrm{L}}\right) \\
\end{array}$}} & \multicolumn{6}{|c|}{ Conditional model } \\
\hline & & & \multicolumn{3}{|c|}{$\begin{array}{l}\text { Offspring born in high-quality habitat } \\
\qquad\left(\mathrm{N}_{\mathrm{H}}\right)\end{array}$} & \multicolumn{3}{|c|}{$\begin{array}{l}\text { Offspring born in low-quality habitat } \\
\qquad\left(\mathrm{N}_{\mathrm{L}}\right)\end{array}$} \\
\hline & Observed & Predicted & Observed & $\begin{array}{l}\text { Predicted, } \\
c=1.01\end{array}$ & $\begin{array}{l}\text { Predicted, } \\
\quad c=5\end{array}$ & Observed & $\begin{array}{l}\text { Predicted, } \\
c=1.01\end{array}$ & $\begin{array}{c}\text { Predicted, } \\
c=5\end{array}$ \\
\hline \multicolumn{9}{|l|}{ Settlement pattern (\%): } \\
\hline Successful QH & 20.0 & $22.4 \pm 7.5$ & 18.2 & $22.4 \pm 7.5$ & $24.8 \pm 7.5$ & 1.8 & 0 & 0 \\
\hline Successful QL & 80.0 & $77.6 \pm 7.5$ & 52.7 & $21.8 \pm 7.7$ & $39.4 \pm 7.6$ & 27.3 & $55.8 \pm 4.2$ & $35.8 \pm 4.6$ \\
\hline $\begin{array}{l}\text { Successful N }(\mathrm{QH}+ \\
\quad \mathrm{QL})\end{array}$ & 100 & 100 & 70.9 & $44.2 \pm 4.2$ & $64.2 \pm 4.1$ & 29.1 & $55.8 \pm 4.2$ & $35.8 \pm 4.6$ \\
\hline \multicolumn{9}{|c|}{ Age of first reproduction (years): } \\
\hline Successful QH & $7.6 \pm .5(11)$ & $7.6 \pm 1.6$ & $7.5 \pm .3(10)$ & $7.6 \pm 1.6$ & $7.5 \pm 1.6$ & $8.0(1)$ & $\ldots$ & $\ldots$ \\
\hline $\begin{array}{l}\text { Successful QL } \\
\text { Successful N (QH + }\end{array}$ & $6.5 \pm .3(44)$ & $3.3 \pm 1.3$ & $6.5 \pm .5(29)$ & $3.3 \pm 1.3$ & $3.1 \pm 1.2$ & $6.5 \pm .2(15)$ & $3.3 \pm 1.3$ & $3.6 \pm 1.3$ \\
\hline QL) & $6.8 \pm .3(55)$ & $4.3 \pm 1.7$ & $6.9 \pm .3(39)$ & $5.5 \pm 1.3$ & $5.3 \pm 1.3$ & $6.6 \pm .7(16)$ & $3.3 \pm 1.3$ & $3.6 \pm 1.3$ \\
\hline
\end{tabular}

Note: Values are given for all offspring combined (unconditional queue model) and for offspring separated by their natal origin (conditional queue model with competitive asymmetries $c=1.01$ or 5). Field data were calculated using all recruits born in 1984-1994 that recruited in the period $1984-2004$ ( $n=$ 55; detection probability of first-time breeders is virtually 1 ). Observed values are given $\pm \mathrm{SE}$, and sample sizes are given in parentheses for age of first reproduction. Predicted values are given $\pm \mathrm{SE}$ and were calculated with the use of 1,000 bootstrapped data sets (see text).

predictions from conditional queuing models that assume that $\mathrm{N}_{\mathrm{H}}$ are competitively superior over $\mathrm{N}_{\mathrm{L}}(c=1.01$; table 1 , top). The quantitative agreement between settlement patterns in the field and model predictions improved even more when we assumed large competitive asymmetries ( $c=5$; table 1 , top). Conditional queuing models did not explain patterns of age of first settlement in the field better than the unconditional model. Age of first settlement in the field also did not differ between offspring born in highand low-quality habitats (6.9 vs. 6.6 years), whereas they were predicted to differ by about 2 years under conditional queuing strategies (table 1, bottom).

\section{Discussion}

\section{General Insights from Queuing Models}

In this study, we first reproduced the main result of EWD: in evolutionary equilibrium, nonbreeders should distribute themselves in such a way over both strategies that the lifetime fitness of both strategies is equal. Delayed reproduction and breeding in low-quality habitat can therefore be part of an adaptive strategy. However, the ESS we derived was substantially different from the ESS in EWD. We think that our evolutionarily stable queuing strategy is more accurate because it is based on a model that combines population dynamics and evolutionary dynamics of life-history strategies in one internally consistent model and also incorporates population regulation. Several other studies have emphasized that evolutionary predictions can strongly depend on assumptions about how populations are regulated (e.g., Mylius and Diekmann 1995; Pen and Weissing 2000). At the same time, our understanding is rather limited as to how populations are regulated and which demographic rates are density dependent in the wild. In this study, we have incorporated population limitation in a simple-but, we think, also biologically plausible-way by fixing the number of suitable territories. Thereby, we do not imply that there is no empty habitat available for additional territories (or that territories cannot split up). However, we do assume that any nonoccupied habitat is of such low quality that it is below the acceptance threshold of nonbreeders; otherwise, it would have been occupied by surplus nonbreeders. The idea of the existence of an acceptance threshold is supported by results from queuing models that investigated the logic of territory choice, which predict a fixed evolutionarily stable threshold quality above which territories are acceptable for nonbreeders (Kokko and Sutherland 1998; Kokko et al. 2001; Pen and Weissing 2001).

We extended the original queue model to allow conditional queuing strategies to evolve (i.e., dependent on natal origin). Thereby, we investigated whether the queue hypothesis can also explain individual variation in settlement strategies. We show that if no competitive asymmetries exist, conditional strategies are unlikely to evolve. However, even very small competitive asymmetries between individuals, which are probably plentiful in nature, will facilitate the evolution of conditional strategies. Thus, individual quality differences in the form of competitive asymmetries strongly affect adaptive queuing decisions at the individual level, emphasizing the importance of investigating the adaptive queuing hypothesis and individual quality hypothesis together instead of separately. This poses the question of to what extent the incorporation of conditional strategies and individual quality differences might also affect predictions from other queuing models 


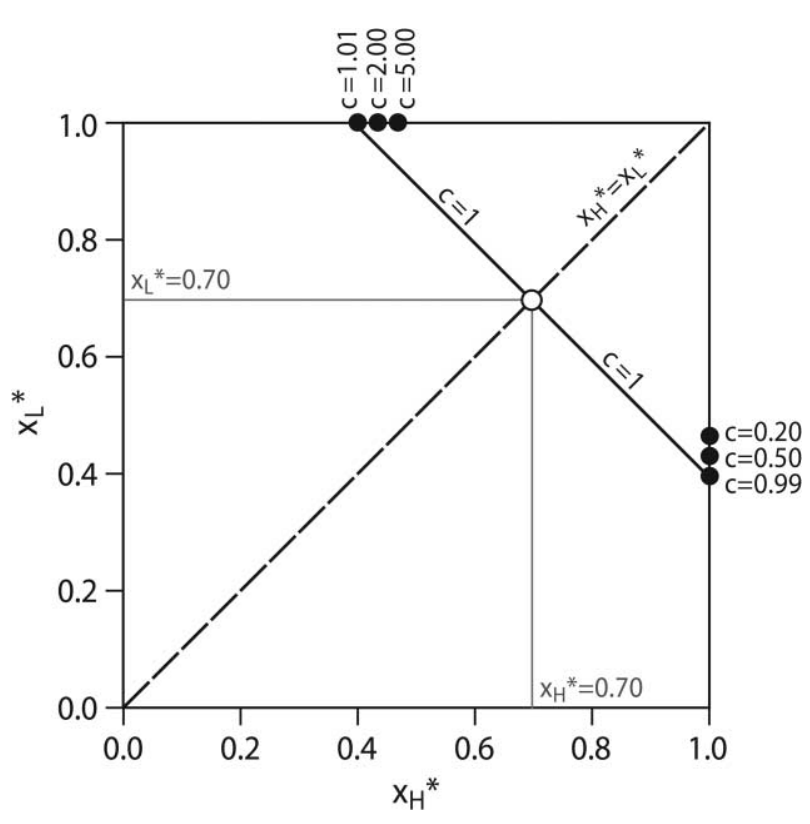

Figure 5: Evolutionarily stable strategy (ESS) values of the strategic queuing variables $x_{\mathrm{H}}^{*}$ and $x_{\mathrm{L}}^{*}$ for various degrees of competitive asymmetry $(c)$. When $c=1$ (no competitive asymmetry), there is a line of equilibria (solid line) that contains the ESS of the unconditional model $\left(x_{\mathrm{H}}^{*}=\right.$ $x_{\mathrm{L}}^{*}=x^{*}=0.70$; open circle). When $c \neq 1$, there always is only one ESS (filled circles), either an ESS where $x_{\mathrm{L}}^{*}$ is a pure strategy (for all $c>1$ ) or an ESS where $x_{\mathrm{H}}^{*}$ is a pure strategy (for all $c<1$ ).

that have ignored such differences for reasons of simplicity. In this study, we incorporated individual quality differences as a function of natal origin. Although effects of early conditions are thought to be very important in nature (Lindström 1999), queuing strategies and competitive asymmetries could also depend on other factors, such as breeding experience. We suspect that other sources of variation in individual quality will also strongly affect adaptive queuing strategies at the individual level but not necessarily at the population level.

\section{Oystercatcher Settlement Patterns: Adaptive Queuing and/or Quality Differences?}

The oystercatcher is an interesting test case of the queue hypothesis because oystercatchers exhibit strong variation in both age of first reproduction and territory quality. Our queuing models were able to predict several striking features of settlement patterns in oystercatchers: (1) age of first reproduction was higher in high-quality habitat than in low-quality habitat; (2) the proportion of first-time recruits that settled in either high- or low-quality habitat was exactly as predicted; and (3) nonbreeders born in lowquality territories virtually never settled in high-quality territories, which is consistent with individuals born in low-quality territories playing a pure QL strategy. These results strongly suggest that oystercatchers make adaptive queuing decisions.

The degree to which individual quality differences affect settlement decisions in oystercatchers is less clear. The agreement between model predictions and oystercatchers' settlement pattern strongly improved when we assumed small differences in competitive abilities. The quantitative agreement between model predictions and field data became even better when we assumed very large differences in competitive abilities. Although it does not seem implausible that very small differences in competitive abilities were overlooked, these results seem to be in sharp contrast with the lack of evidence for strong differences in competitive abilities in oystercatchers (EWD; Bruinzeel et al. 2006). Possibly, the discrepancy is not as strong as we think but might be (partially) caused by the difficulty of measuring differences in competitive abilities between nonbreeders in the field. Observation of physical fights between nonbreeders are rare, and outcomes of ritualized fights are strongly site dependent in oystercatchers (Heg et al. 2000). Consequently, both studies had to rely on indirect measures of competitive abilities over breeding territories, such as morphology and fights on feeding grounds or in captivity. Clearly, evidence from more direct measures of differences in competitive abilities over breeding territories between nonbreeders is crucial. In fact, given that individuals born in high-quality habitat are $10 \%$ heavier at fledging than individuals born in low-quality habitat (van de Pol et al. 2006), we would not be surprised if future research indicates that natal origin does indeed influence competitive abilities in oystercatchers. Although, at the moment, this part of the puzzle remains unsolved, it does point out a more general problem. Our queuing models are very sensitive to assumptions about competitive asymmetries, while detecting such differences in the wild is likely to be difficult in many species (i.e., how to measure parameter $c$ ).

Although the observation that age of first reproduction is higher in high-quality habitat than in low-quality habitat was qualitatively in agreement with the queuing models, the difference in age of first reproduction in the wild was 3 years less than predicted. This discrepancy was because age of first reproduction of oystercatchers in low-quality territories was higher than predicted. Two alternative individual quality hypotheses might explain this discrepancy. First, the high age of first settlement in low-quality habitat in the field might suggest that nonbreeders that queue for low-quality habitat reach sexual maturity later in life than others. Similarly, if queuing for low-quality territories is less costly than queuing for high-quality territories $\left(\mu_{\mathrm{QL}}<\mu_{\mathrm{QH}}\right)$, this would also increase the predicted age of 
first settlement in low-quality habitat. Second, except for differences in natal background, we implicitly assumed that all queuing individuals had an equal chance of acquiring the territory they were queuing for ("randomorder service"). However, queues of oystercatchers probably more strongly resemble strict queuing ("first in, first out"), because social dominance seems to improve gradually during queuing and subsequently facilitates territory acquisition (Heg et al. 2000; Bruinzeel and van de Pol 2004). For example, oystercatchers with breeding experience have a higher annual settlement probability than inexperienced nonbreeders (Bruinzeel 2007). Incorporating mechanisms into our models that structure queues into hierarchies based on breeding experience is likely to result in a predicted age of first settlements in low-quality habitat that is closer to those in the field.

\section{Population Consequences of Queuing Strategies}

Although we applied our queuing models to period 1 only, period 2 provides some useful insights into the consequences of queuing processes at the population level. During period 2, the number of low-quality territories halved, while the number of high-quality territories and, most interesting, also the number of nonbreeding oystercatchers remained fairly stable (fig. 2). This pattern of population decline supports the idea that nonbreeders can discriminate between the quality of different habitats because vacancies in high-quality habitat were reoccupied, while many vacancies in low-quality habitat remained empty in period 2. Furthermore, the abandonment of low-quality territories in the presence of many nonbreeders strongly suggests that these nonbreeders preferred to queue for a high-quality territory instead of settling in vacated lowquality territories. But why would surplus nonbreeders choose not to occupy vacant low-quality territories in period 2, while they were willing to do so in period 1 ? We think that this change in queuing behavior results from a general decline in environmental quality from period 1 to period 2, caused by a drop in food supplies (Bruinzeel and van de Pol 2003). In period 1, the reproductive value of breeding in a low-quality territory was still slightly higher $(10 \%)$ than the reproductive value of nonbreeders. When in period 2 fecundity dropped as a result of low hatching success, nonbreeders probably perceived the value of many low-quality territories to be reduced below the threshold value for territory acceptance and therefore stopped queuing for them $\left(v_{\mathrm{L}}<v_{\mathrm{N}}\right.$; see Kokko and Sutherland 1998; Pen and Weissing 2001). This idea is further supported by recent observations of pairs that abandoned their low-quality territory to become nonbreeders, while there were no indications for widowing, divorce, or takeovers (M. van de Pol, personal observation). Consequently, population productivity in period 2 was probably lower than it would have been had nonbreeders reoccupied all empty low-quality habitat. Thus, although queuing behavior might be optimal for individuals, it generally is not for the population (e.g., Kokko et al. 2001). Therefore, for understanding both life-history decisions and population dynamics, studying the behavior of individuals that do not (yet) reproduce might be as important as studying the behavior of the reproductive part of the population.

\section{Acknowledgments}

We thank Natuurmonumenten for allowing us to work in the national park. This research would have been impossible without the continuous support of R. Drent and J. Tinbergen. We thank all the people who contributed to the fieldwork over the years, especially L. Bruinzeel, B. Ens, J. Hulscher, M. Kersten, and K. Oosterbeek. We thank L. Bruinzeel, B. Ens, and S. Verhulst for discussion. Comments by two reviewers greatly improved the manuscript. D. Visser drew the figures. M.v.d.P. was supported by the Breedtestrategie program "Dispersal and Adaptation" (University of Groningen) and by a Rubicon fellowship of the Netherlands Organization for Scientific Research (NWO; grant 825.06.032). D.H. was supported by NWO grant SLW-805.30.164 and is currently supported by Swiss National Science Foundation grant 3100A0-108473.

\section{Literature Cited}

Bruinzeel, L. W. 2007. Intermittent breeding as a cost of site fidelity. Behavioral Ecology and Sociobiology 61:551-556.

Bruinzeel, L. W., and M. van de Pol. 2003. Population decline of oystercatchers breeding on Schiermonnikoog. Limosa 76:25-26. [In Dutch.]

- 2004. Site attachment of floaters predicts success in territory acquisition. Behavioral Ecology 15:290-296.

Bruinzeel, L. W., M. van de Pol, and C. Trierweiler. 2006. Competitive abilities of oystercatchers (Haematopus ostralegus) occupying territories of different quality. Journal of Ornithology 147:457-463.

Caswell, H. 2001. Matrix population models: construction, analysis and interpretation. 2nd ed. Sinauer, Sunderland, MA.

Charnov, E. L. 1991. Evolution of life-history variation in female mammals. Proceedings of the National Academy of Sciences of the USA 88:1134-1137.

East, M. L., and H. Hofer. 2000. Male spotted hyenas (Crocuta crocuta) queue for status in social groups dominated by females. Behavioral Ecology 12:558-568.

Ekman, J., S. Eggers, M. Griesser, and H. Tegelstrom. 2001. Queuing for preferred territories: delayed dispersal of Siberian jays. Journal of Animal Ecology 70:317-324.

Ens, B. J., M. Kersten, A. Brenninkmeijer, and J. B. Hulscher. 1992. Territory quality, parental effort and reproductive success of oystercatchers (Haematopus ostralegus). Journal of Animal Ecology 61:703-715.

Ens, B. J., F. J. Weissing, and R. H. Drent. 1995. The despotic dis- 
tribution and deferred maturity: two sides of the same coin. American Naturalist 146:625-650.

Fretwell, S. D. 1972. Populations in seasonal environment. Princeton University Press, Princeton, NJ.

Heg, D., B. J. Ens, T. Burke, L. Jenkins, and J. P. Kruijt. 1993. Why does the typically monogamous oystercatcher (Haematopus ostralegus) engage in extra-pair copulations? Behaviour 126:247-289.

Heg, D., B. J. Ens, H. P. van der Jeugd, and L. W. Bruinzeel. 2000. Local dominance and territorial settlement of nonbreeding oystercatchers. Behaviour 137:473-530.

Heg, D., L. W. Bruinzeel, and B. J. Ens. 2003. Fitness consequences of divorce in the oystercatcher, Haematopus ostralegus. Animal Behaviour 66:175-184.

Heg, D., Z. Bachar, and M. Taborsky. 2005. Cooperative breeding and group structure in the Lake Tanganyika cichlid Neolamprologus savoryi. Ethology 111:1017-1043.

Kokko, H., and J. Ekman. 2002. Delayed dispersal as a route to breeding: territorial inheritance, safe havens, and ecological constraints. American Naturalist 160:468-484.

Kokko, H., and R. A. Johnstone. 1999. Social queuing in animal societies: a dynamic model of reproductive skew. Proceedings of the Royal Society B: Biological Sciences 266:571-578.

Kokko, H., and W. J. Sutherland. 1998. Optimal floating and queuing strategies: consequences for density dependence and habitat loss. American Naturalist 152:354-366.

Kokko, H., J. Lindström, R. V. Alatalo, and P. T. Rintamäki. 1998. Queuing for territory positions in the lekking black grouse (Tetrao tetrix). Behavioral Ecology 9:376-383.

Kokko, H., W. J. Sutherland, and R. A. Johnstone. 2001. The logic of territory choice: implications for conservation and source-sink dynamics. American Naturalist 157:459-463.

Krüger, O. 2005. Age at first breeding and fitness in goshawk Accipiter gentilis. Journal of Animal Ecology 74:266-273.

Lindström, J. 1999. Early development and fitness in birds and mammals. Trends in Ecology \& Evolution 14:343-349.

Maynard Smith, J., and G. A. Parker. 1976. The logic of asymmetric contests. Animal Behaviour 24:159-175.

Mitchell, J. 2005. Queue selection and switching by false clown anemonefish Amphiprion ocellaris. Animal Behaviour 69:643-652.

Mylius, S. D., and O. Diekmann. 1995. On evolutionary stable life histories, optimization and the need to be specific about density dependence. Oikos 74:218-224.

Newton, I. 1998. Population limitation in birds. Academic Press, London.

Oli, M. K., G. R. Hepp, and R. A. Kennamer. 2002. Fitness consequences of delayed maturity. Evolutionary Ecology Research 4: 563-576.

Pen, I., and F. J. Weissing. 2000. Towards a unified theory of cooperative breeding: the role of ecology and life history re-examined. Proceedings of the Royal Society B: Biological Sciences 267:2411-2418.

2001. Optimal floating and queuing strategies: the logic of territory choice. American Naturalist 155:512-526.

Poston, J. P. 1997. Dominance, access to colonies, and queues for mating opportunities by male boat-tailed grackles. Behavioral Ecology and Sociobiology 41:89-98.

Safriel, U. N., B. J. Ens, and A. Kaiser. 1996. Rearing to independence. Pages 219-250 in J. D. Goss-Custard, ed. The oystercatcher: from individuals to populations. Oxford University Press, Oxford.

Schwagmeyer, P. L., and G. A. Parker. 1987. Queuing for mates in the thirteen-lined ground squirrel. Animal Behaviour 35:10151025.

Stearns, S. C. 1992. The evolution of life histories. Oxford University Press, Oxford.

Taylor, P. D. 1990. Allele-frequency change in a class-structured population. American Naturalist 135:95-106.

van de Pol, M., L. W. Bruinzeel, D. Heg, H. P. van der Jeugd, and S. Verhulst. 2006. A silver spoon for a golden future: long-term effects of natal origin on fitness prospects of oystercatchers. Journal of Animal Ecology 75:616-626.

Voigt, C. C., and W. J. Streich. 2003. Queuing for harem access in colonies of the greater sac-winged bat. Animal Behaviour 65:149156.

Wiley, R. H., and K. Rabenold. 1984. The evolution of cooperative breeding by delayed reciprocity and queuing for favorable positions. Evolution 38:609-621.

Zack, S., and B. J. Stutchbury. 1992. Delayed breeding in avian social systems: the role of territory quality and "floater" tactics. Behaviour 123:194-219.

Associate Editor: Peter D. Taylor Editor: Michael C. Whitlock 